This item was submitted to Loughborough's Research Repository by the author.

Items in Figshare are protected by copyright, with all rights reserved, unless otherwise indicated.

\title{
Intermodulation distortion in a photoconductive microwave switch
}

PLEASE CITE THE PUBLISHED VERSION

https://doi.org/10.1109/LAPC.2016.7807508

PUBLISHER

(c) IEEE

VERSION

AM (Accepted Manuscript)

LICENCE

CC BY-NC-ND 4.0

REPOSITORY RECORD

Kowalczuk, Emma K., C.J. Panagamuwa, and R.D. Seager. 2019. "Intermodulation Distortion in a Photoconductive Microwave Switch". figshare. https://hdl.handle.net/2134/24325. 


\section{Intermodulation Distortion in a Photoconductive Microwave Switch}

\author{
Emma K. Kowalczuk \\ Jaguar Land Rover \\ Electrical Engineering, Banbury Road, Gaydon \\ Warwickshire, U.K.
}

\author{
Chinthana J. Panagamuwa and Rob D. Seager \\ Wolfson School of Mechanical, Electrical and \\ Manufacturing Engineering, \\ Loughborough University \\ c.j.panagamuwa@lboro.ac.uk
}

\begin{abstract}
Single-tone harmonic and two-tone intermodulation distortion measurements are presented for a photoconductive microwave switch. The switch consists of a lightly doped die of silicon mounted over a gap in a transmission line. The switch is controlled via near infra-red light delivered by a fiber optic cable. Whilst under constant illumination, the third order intercept for a $2 \mathrm{GHz} \mathrm{CW}$ signal is extrapolated to be $\mathbf{7 4}$ $\mathrm{dBm}$. Under a $1 \mathrm{MHz}$ spaced two-tone signal, the extrapolated intermodulation intercept point is $58 \mathrm{dBm}$.
\end{abstract}

Keywords - Photoconductive switch, optical control, fiber optic, silicon, linearity, intermodulation, harmonic distortion, microwave switch, reconfigurable antenna

\section{INTRODUCTION}

The design of reconfigurable antennas and circuits is actively being researched in order to increase functionality of wireless systems. The form factor of the antennas can be greatly reduced as parts of the geometry are reused and so a compact and portable solution is possible. In order to achieve this goal of frequency, pattern or polarization tuning, a robust switching mechanism that has minimal effect on performance is a key requirement. The traditional PIN diode switch has the disadvantages of the need to incorporate metallic biasing lines and is non-linear in behavior. In contrast, optically controlled microwave switches offer the advantage of being electromagnetically transparent at RF frequencies. The device performance need not be compromised by the presence of metallic biasing lines [1].

The use of photoconductive switches has been proven in a variety of applications. In principle, silicon dice can be illuminated to alter operating frequency, with a similar approach used to also reconfigure beam direction [2] and polarization [3]. Optically controlled switches have also been reported in attenuators [4] and filters [5]. Particularly in the case of miniature filter design, the design of the biasing lines needed to operate these components must be considered carefully and can sometimes be a limitation on the size and layout of the device. In our research, a fiber optic cable is used to deliver illumination which is generated in a remote location away from the RF circuitry.

The linearity and power handling of switch mechanisms is particularly relevant in high power RF applications. Due to the $\mathrm{p}-\mathrm{n}$ junction in PIN and varactor diodes, these components are non-linear and produce distortion. PIN diodes are mainly constructed to exhibit fast switching speeds, which require a small intrinsic layer and a small carrier lifetime. These values have a direct effect on the tendency to retain a quiescent level of stored charge, and hence create distortion [6].

The effect of distortion of the switch mechanism has been investigated by Yong et al. [7]. The example of a varactor diode in a reconfigurable patch antenna is used to demonstrate the non-linear effects on antenna performance. From the perspective of electromagnetic interference (EMI) conformity issues, Kalialakis et al. reported on the importance of harmonic performance for a quarter-wavelength microstrip antenna switched via varactor diode [8]

Improving the linearity of diode switches has been a focus of research, resulting in configurations of switches such as reported by Huang et al. [9]. A third order intermodulation product with respect to the output power (OIP3) of $56 \mathrm{dBm}$ is reported in this instance for a configuration of four diodes. The principle is based on a back-to back configuration resulting in cancellation of distortion currents. The downside of this approach is that no two devices will exhibit the same distortion so cancellation will not be absolute. Multiple devices are required which increase complexity and footprint of the switching mechanism.

Conversely, MEMS switches demonstrate high linearity, as there are no semiconductor junctions in these components. Typical OIP3 values are tested to above $65 \mathrm{dBm}$ for a $1 \mathrm{MHz}$ tone spacing at $1 \mathrm{GHz}$ [10]. These devices have been incorporated into reconfigurable circuits and antennas as is demonstrated by a monolithic integration approach by Crusats et al. [11]. However their power handling capabilities can be limited by self actuation.

The photoconductive switch presented in this paper does not require a $\mathrm{p}-\mathrm{n}$ junction to function. The switch does not incorporate moving parts and so should be less expensive to manufacture as compared to MEMS and not suffer failure due to self-actuation under high power signals.

The focus of this paper is to report on both the harmonic single tone linearity and two tone intermodulation distortion. The single tone linearity is determined in this instance by the appearance of undesired harmonics at multiples of the fundamental test frequency. It is reported in terms of the Third Order Intercept (TOI) value. Two tone intermodulation 
distortion is measured as the distortion caused within the switch when a two-tone signal is passed through the switch. This is determined by the Third Order Intermodulation Intercept Point, IP3 with respect to either the input power, IIP3 or the output power OIP3.

The use of photoconductive switches in reconfigurable devices has clear advantages. This research highlights the potential for the photoconductive switch to be used in higher power systems, with minimal detriment to performance.

\section{SWITCH OPERATION}

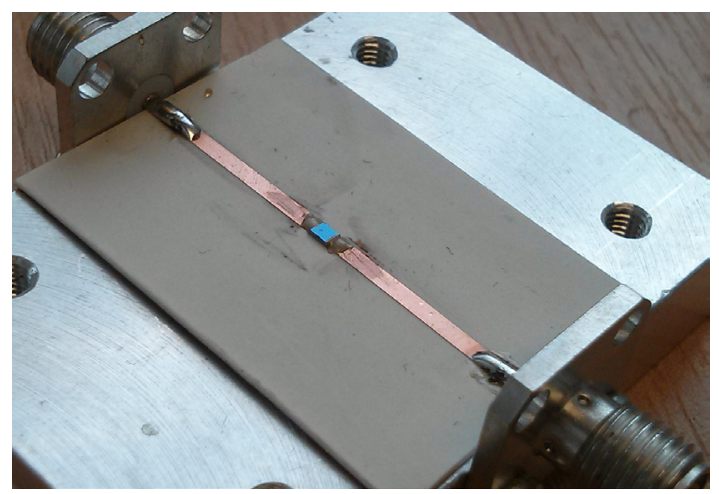

Fig. 1. Photoconductive microwave switch consisting of a gap in transmission line bridged by a $1 \mathrm{~mm} \times 1 \mathrm{~mm} \times 0.3 \mathrm{~mm}$ die of silicon

The photoconductive switches consist of a die of phosphorus doped silicon, resistivity $>6000 \Omega \mathrm{m}$, attached between two lengths of copper microstrip line, Fig. 1, similar to the kind used by Panagamuwa et al. [12]. When illuminated by near infra-red light, electrons in the silicon are promoted from the valence band to the conduction band. Electron-hole pairs are created, hence making the silicon conductive. The switch is in its closed (ON state) when illuminated which allows the RF signal to propagate through the silicon.

Once the light is removed the electron-hole pairs recombine within the silicon. Any free electrons are swept out of the silicon and the switch becomes open (OFF state), behaving as an insulator and blocking the RF signal through the silicon. The switch does not incur any degradation in this process.

The transmission line topology has been optimized in a previous study [13]. The switch is designed on $0.52 \mathrm{~mm}$ thick Rogers 3003 substrate which is mounted on an aluminum ground plane. The gap in transmission line is $0.26 \mathrm{~mm}$, and the silicon die measuring $1 \mathrm{~mm} \times 1 \mathrm{~mm} \times 0.3 \mathrm{~mm}$ is attached directly over the gap using silver epoxy, (Fig. 1).

The switch is controlled using a laser diode system. Illumination from the remotely located laser diode is coupled into a $1 \mathrm{~mm}$ diameter fused silica fiber optic cable which is positioned directly over the silicon die. The wavelength of light used in this study is $980 \mathrm{~nm}$ and is delivered as a continuous wave with radiant flux of $200 \mathrm{~mW}, 50 \mathrm{~mW}$ or 30 $\mathrm{mW}$. The ambient temperature for all experiments is $24^{\circ} \mathrm{C}$.
Note that all linearity tests are performed under cold switching conditions.

\section{HARMONIC TESTING}

\section{A. Single-tone Linearity}

A $2 \mathrm{GHz}$ input signal between 26-34 dBm was generated using a signal generator. A low pass filter was added in between the RF source and the photoconductive switch to reduce harmonics generated by the signal generator. The power levels used ensure the switch is still driven in the linear region. The power at the fundamental frequency of $2 \mathrm{GHz}$ is recorded at the input of the switch using a coupler whilst the output is recorded using a spectrum analyzer, Advantest R3132. The harmonic content at the output of the switch is also recorded. The harmonics appear at $2 \mathrm{f}_{0}$ and $3 \mathrm{f}_{0}$, which correspond to $4 \mathrm{GHz}$ and $6 \mathrm{GHz}$.

This data is used to extrapolate the second and third order intercept (SOI and TOI) of the switch. The single tone linearity of this type of switch has been reported previously [14], where the experimental setup is shown in more detail, however results for an improved topology of the switch are reported here. In this instance, three test cases are investigated; $30 \mathrm{~mW} 50 \mathrm{~mW}$ and $200 \mathrm{~mW}$ of radiant flux are used to control the photoconductive switch.

\section{B. Single-tone Results}

Third Order intercept (TOI) is a theoretical value where the fundamental power out equals the third order harmonic power. The third order harmonic occurs at $6 \mathrm{GHz}$, a line of best fit is

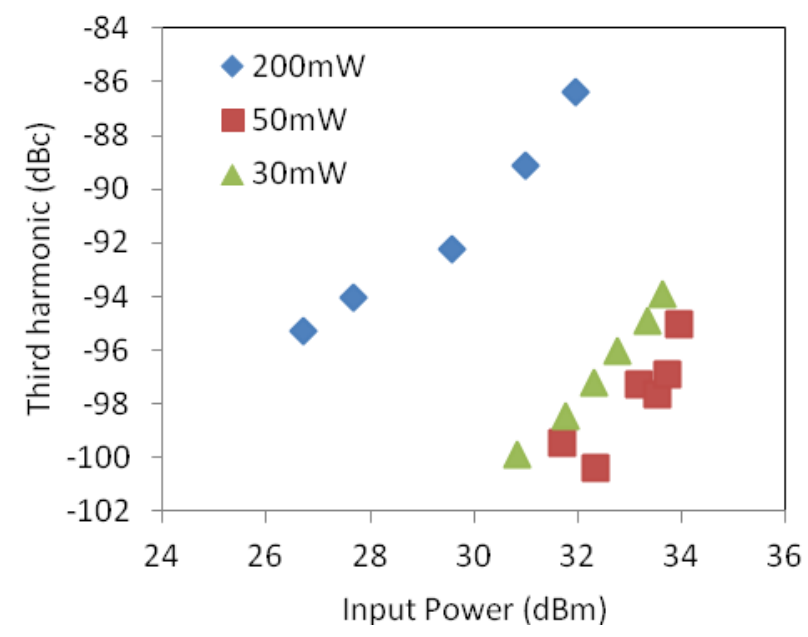

Fig. 2. Measured third order harmonics expressed in terms of the fundamental carrier for 30,50 and $200 \mathrm{~mW}$ of radiant flux.

extrapolated from the measured data. The gradient of this line agrees well with the theoretical value of three that is expected.

For the maximum radiant flux of $200 \mathrm{~mW}$ the value for TOI is $74 \mathrm{dBm}$. The value for Second Order Intercept which has an expected slope gradient of two is $105 \mathrm{dBm}$, Fig. 2. These values indicate the switch is linear in nature. 
Fig. 2 also indicates the level of harmonic content for the $30 \mathrm{~mW}$ and $50 \mathrm{~mW}$ illumination scenarios, expressed in $\mathrm{dB}$ below the fundamental. The TOI when $30 \mathrm{~mW}$ of radiant flux is applied is $77 \mathrm{dBm}$ and for $50 \mathrm{~mW}$ is $78 \mathrm{dBm}$. This demonstrates that the linearity does not change significantly under varying radiant flux.

\section{INTERMODULATION DISTORTION}

\section{A. Two-tone Linearity Setup}

Typically, only a broadband system is susceptible to single tone harmonic distortion because harmonics usually occur well away from the input frequency of the signal. When utilizing the switch in a narrow band application, the intermodulation distortion is also of concern as the distortion normally occurs in the band of use.

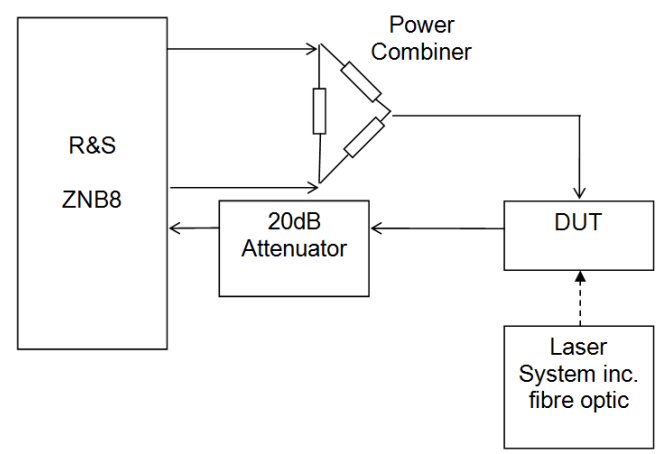

Fig. 3 Test setup for intermodulation Measurements

The Rohde and Schwarz ZNB8 was used to perform the twotone linearity characterization. Two internal signal generators were used to generate the two tones based around a centre frequency of $2 \mathrm{GHz}$ and spaced $1 \mathrm{MHz}$ apart. The signals were combined using a power combiner and connected to the switch, as is shown in Fig. 3 and 4. A $20 \mathrm{~dB}$ attenuator was placed on the input to the ZNB8 in order to reduce distortion at the receiver.

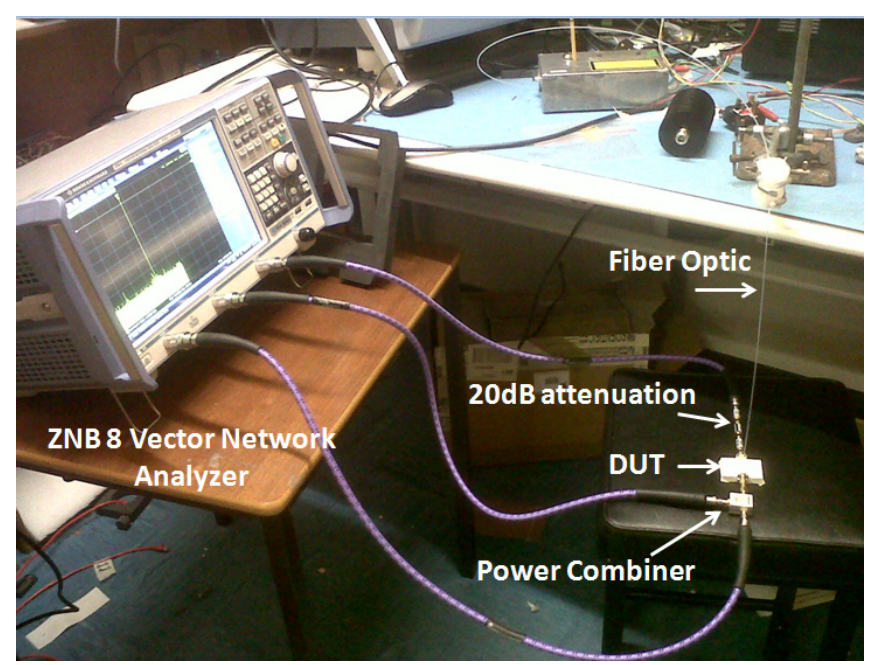

Fig. 4 Two-tone IM Distortion measurement setup in the process of capturing data. Equipment was setup to allow minimum noise floor.
Power calibration of the system was performed in order to remove cable losses and the effect of the attenuator on the results.

The power was swept between $0-6 \mathrm{dBm}$ and the level of the intermodulation product was recorded at $2 f_{1}-f_{2}$ and $2 f_{2}-f_{1}$. $\mathrm{f}_{1}$ refers to the frequency of the lower tone $(1.9995 \mathrm{GHz})$ and $\mathrm{f}_{2}$ is the frequency of the upper tone $(2.0005 \mathrm{GHz})$. This testing was performed whilst the switch was illuminated by a radiant flux of $50 \mathrm{~mW}$.

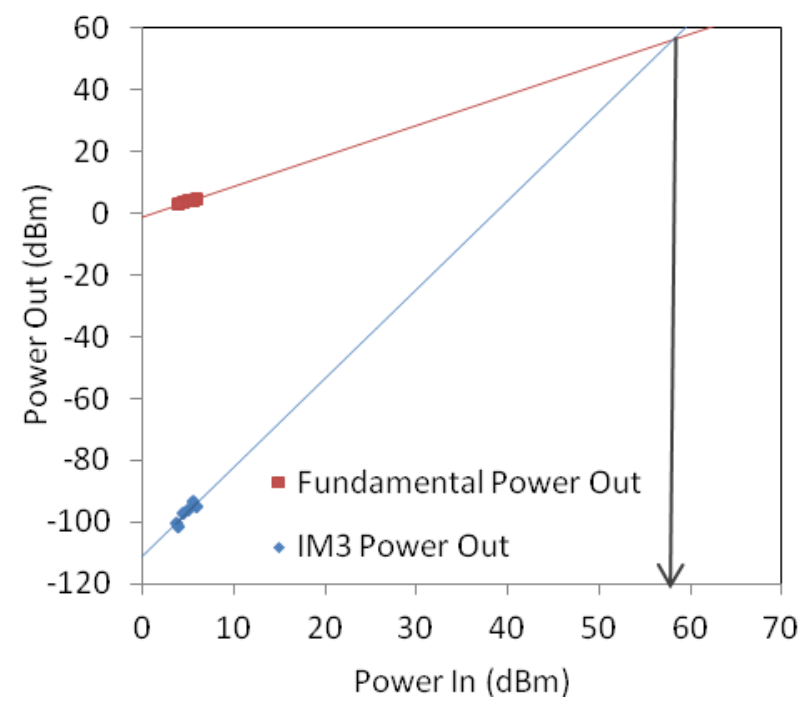

Fig. 5. Measured fundamental $(2 \mathrm{GHz})$ and third order intermodulation (lower $2 \mathrm{f}_{1}-\mathrm{f}_{2}$ ) for a photoconductive switch under $50 \mathrm{~mW}$ of radiant flux.

\section{B. Two-tone Linearity Results}

Similarly to the single tone harmonics, the measured intermodulation distortion is expected to increase at a ratio of $3: 1$. Results agree with the theoretical gradient of 3 . With an input of $6 \mathrm{dBm}$, the third order intermoduation product occurs at $-96 \mathrm{dBm}$ for the lower-tone. The extrapolated value for intercept point between distortion and fundamental is $58 \mathrm{dBm}$ with reference to the input power, Fig.5. Distortion values for the upper tone were similar to the lower tone as expected. Note, for larger tone spacing the value of distortion is expected to be decreased further. The source of possible distortion will be discussed in the following section.

\section{DISCUSSION}

Many types of RF switches exist and have been optimized for different purposes. In general, the photoconductive switch has better linearity values when compared to commercially available PIN switches. The advantage to the photoconductive switch is that no metallic biasing lines are required in the plane of the RF circuitry, thus eliminating EM interference.

A possible reason as to why photoconductive switches create less distortion to the input signal is their structure. The junction within PIN diodes creates a difference in capacitance and non linear resistance as the signal propagates through the 
switch; no such junction exists in the type of photoconductive switch presented in this study.

There are a number of other factors which may affect the linearity of a photoconductive switch. For PIN diodes, the size of the intrinsic layer and the purity both affect distortion levels within the switch. Likely causes of distortion in the photoconductive switch arise from the differing conductivity and permittivity profiles generated within the silicon when illuminated,[15] and [16]. The changes in permittivity and loss tangent in the silicon may cause distortion in the incoming RF signal as it propagates through the silicon.

The switch discussed in this work has been processed to improve its quality in terms of carrier lifetime, meaning distortion is minimized. The silicon dimensions have been matched to the fiber dimensions, hence a more uniform lateral conductivity profile is created across the length of the silicon. A longer wavelength of light also increases the penetration depth producing a more uniform conductivity profile throughout the depth of the silicon. The impurities in the silicon are kept to a minimum as it has been fabricated using the float-zone method. The doping concentration is also relatively low; meaning the distortion within the switch can be kept to a minimum.

The high carrier lifetime of the silicon also improves distortion performance. For semiconductors with a larger carrier lifetime, the capacitance value within the device remains more constant and hence distortion is less prevalent. The quasi-steady state photoconductance method was used to monitor the minority carrier lifetime in the complete silicon wafer used to produce the photoconductive switch. This value is measured as $\sim 600 \mu \mathrm{s}$, and directly affects the carrier concentration in the illuminated silicon. This initial value is just an indication of the actual carrier lifetime within the switch as when the silicon is diced, the damage caused at the edges will reduce the carrier lifetime. Measured rise and fall times of the switch are reported in previous work by Kowalczuk et al. [17]. The silicon has been designed to have a high carrier lifetime to improve conductivity and has the added benefit of producing reduced distortion.

\section{CONCLUSION}

Figures of merit for power handling ability such as transmission characteristics, single-tone and two-tone linearity have been presented for a photoconductive microwave switch. The low insertion loss of $0.55 \mathrm{~dB}$ at $2 \mathrm{GHz}$ suggests that high RF powers can be handled by the switch with small ohmic losses, potentially being handled through a well-designed package. Single-tone third order intercept point is extrapolated to be $74 \mathrm{dBm}$. Two-tone third order intercept point is extrapolated to be $58 \mathrm{dBm}$ for a tone spacing of $1 \mathrm{MHz}$. The switch demonstrates a highly linear behavior with the added benefit that no metallic biasing lines are required.

\section{ACKNOWLEDGMENT}

The authors would like to thanks Rohde and Schwarz for the use of their test equipment. The authors would also like to acknowledge Rogers for the use of their substrate in this study.

\section{REFERENCES}

[1] A. M. Yadav, C. J. Panagamuwa, and R.D. Seager, "Investigating the effects of control lines on a frequency reconfigurable patch antenna," Loughborough Antennas and Propagation Conference (LAPC), Nov. 2010

[2] S. Pendharker, R. K. Shevgaonkar, and A. N. Chandorkar. "Optically controlled frequency-reconfigurable microstrip antenna with low photoconductivity." IEEE Antennas and Wireless Propagation Letters 13, 99-102, 2014

[3] S. Hansheng, I. Shoaib, C. Xiadong and T. Kreouzis, "Optically Tuned Polarisation Reconfigurable Antenna," IEEE Asia-Pacific Conference on Antennas and Propagation (APCAP), pp. 265-266, 27-29 Aug. 2012

[4] J. R. Flemish, and R. L. Haupt, "Optimisation of a photonically controlled microwave switch and attenuator," IEEE transactions on microwave theory and techniques, vol. 58, No. 10, pp. 2582-2588, 2010.

[5] K. Rabbi, L. Athukorala, C. Panagamuwa, J. C. Vardaxoglou and D. Budimir, "Highly linear microstrip wideband bandpass filter with switchable notched band for wireless applications," Microwave and Optical Technology Letters, Vol.55, no. 6 pp.1331-1335, 2013

[6] MACOM technology Solutions "Design with PIN Diodes" AG132 Application Note. Last accessed 2013

[7] S. Yong, "Design and analysis of pattern null reconfigurable antennas," Ph.D. dissertation, Dept. Elect. and Comp. Eng., Illinois Univ., UrbanaChampaign, IL, 2012.

[8] C. Kalialakis and P. S. Hall, "Analysis and experiment on harmonic radiation and frequency tuning of varactor-loaded microstrip antennas," IET Microw. Ant. and Prop., vol. 1, no. 2, pp. 527-535, 2007.

[9] C. Huang et al.., "Ultra linear low-loss varactor diode configurations for adaptive RF systems," IEEE transactions on microwave theory and techniques, Vol. 57, No. 1, pp. 205-215, January 2009.

[10] Radant MEMS "RMSW100HP High Power MEMS," www.radant.com, last accessed Jan. 2015

[11] G. Crusats, L. Jofre, and N. Biyikli, "RF MEMS Integrated Frequency Reconfigurable Annular Slot Antenna," IEEE Trans. Antennas Propagat. Vol. 58, No. 3, pp. 626-632, March 2010

[12] C.J. Panagamuwa, A. Chauraya and J.C. Vardaxoglou, "Frequency and beam reconfigurable antenna using photoconducting switches" IEEE Trans. Antennas Propagat., vol. 54, no.2, pp. 449-454, 2006.

[13] E. K. Kowalczuk, , R. D. Seager, C. J. Panagamuwa, K. Bass and J. C. Vardaxoglou, "Optimising the performance of an optically controlled photoconductive microwave switch," Loughborough Antennas and Propagation Conference (LAPC), Nov. 2012

[14] E. K. Kowalczuk, C. J. Panagamuwa, R. D. Seager, and J. C. Vardaxoglou, "Characterising the linearity of an optically controlled photoconductive microwave switch," Loughborough Antennas and Propagation Conference (LAPC), Nov. 2010

[15] W. Platte "Effective photoconductivity and plasma depth in optically quasi-CW controlled microwave switching devices" IEE Proceedings J, Optoelectronics, Vol.135, Issue 3, pp. 251 - 254, June 1988

[16] C. Lee, P. Mak, A. Defonzo "Optical control of millimeter-wave propagation in dielectric waveguides," IEEE Journal of Quantum Electronics, Vol. 16, Issue 3, pp.277-288, March 1980, Vol. 14, No. 18, August 1978

[17] E. K. Kowalczuk, C. J. Panagamuwa, and R. D. Seager, "Design and operation influences regarding rise and fall time of a photoconductive microwave switch," Loughborough Antennas and Propagation Conference (LAPC), pp. 149-154, Nov. 2013. 